\title{
Detection of free core nutation
} resonance variation in Earth tide from global superconducting gravimeter observations

\author{
Xiaoming Cui ${ }^{1 *}\left(\mathbb{D}\right.$, Heping Sun ${ }^{1,2}$, Jianqiao Xu' ${ }^{1}$, Jiangcun Zhou ${ }^{1}$ and Xiaodong Chen ${ }^{1}$
}

\begin{abstract}
In order to verify the time variability of free core nutation (FCN) period, global superconducting gravimeter (SG) observations were analyzed based on synthetic test data. The gravity data series were synthesized to check the detectability of resonance variation caused by FCN period change. The tests indicate that the discrepancy between the FCN periods determined by SG and VLBI observations is caused by the high correlation between the FCN parameter and the amplitude factor of the $\psi 1$ wave. The K1 wave is more sensitive to the FCN period change than other diurnal waves. The limit of the standard deviation of the K1 wave is found for more precisely observing the FCN period change. Tidal parameters of diurnal waves estimated from long series of 20 global SG stations were analyzed. A common variation trend is found in the amplitude factor of both $\mathrm{K} 1$ and $\psi 1$ waves in all 8 stations above the limit, which indicates the FCN period may be not so stable in time. Furthermore, the variation in the $\mathrm{K} 1$ and $\psi 1$ waves constrains the FCN period change to between 2.5 and 4 sidereal days, which also agrees with the possible variation from the current VLBI and SG observations.
\end{abstract}

Keywords: Free core nutation, Superconducting gravimeter, Earth tide, Temporal variation

\section{Introduction}

The interaction between the elliptical liquid core and the solid mantle, due to the misalignment of their instantaneous rotation axes, produces one of the Earth's rotational modes, called free core nutation (FCN) in the celestial reference system and nearly diurnal free wobble (NDFW) in the terrestrial reference system.

The FCN causes resonance enhancement to nearby Earth tidal waves/nutation terms in the diurnal/annual frequency band. This enhancement offers the most commonly used approach to determine the characteristics (such as period and quality factor) of the FCN based on high precision tide and nutation observations from superconducting gravimeter (SG) and VLBI (very-longbaseline interferometry) techniques, respectively.

\footnotetext{
*Correspondence: cxm@asch.whigg.ac.cn

1 State Key Laboratory of Geodesy and Earth's Dynamics, Institute of Geodesy and Geophysics, Chinese Academy of Sciences,

Wuhan 430077, China

Full list of author information is available at the end of the article
}

Numerous previous works have estimated the FCN period using either SG or VLBI observation (Ducarme et al. 2007; Rosat and Lambert 2009). It should be pointed out that the FCN period from VLBI observations mentioned in this paper is the one obtained based on the resonant enhancement of some nutation components in the vicinity of the FCN frequency. In Mathews et al. (2002), the FCN period estimated with this approach equals to 431.4 sidereal days (SDs) which is adopted in the IAU 2006/2000 precession-nutation model. Besides, FCN signal is clearly seen in the celestial pole offsets determined from VLBI observations since 1984. But this signal shows significant time-varying characteristics in terms of period, phase and amplitude. The period determined from direct observation can differ by several days depending on the applied method of data reduction and analysis (Chao and Hsieh 2015; Zhou et al. 2016), which is probably due to the convolution with excitation sources. The difference between the resonant period and the observed period is still an open question. What we focused in this study is the resonant period which is 
relatively stable around 430 SDs. However, this value can fluctuate by a few days when using observations in a different time span, which raises the issue as to whether a temporal variation exists in the FCN period. Considering that the FCN period closely relates to the core-mantle boundary $(\mathrm{CMB})$, its variation might reflect certain changes at the $\mathrm{CMB}$, providing a good reference for deep Earth probing. This issue has been discussed in a few previous studies. Most researchers (Roosbeek et al. 1999; Hinderer et al. 2000; Lambert and Dehant 2007; Vondrák and Ron 2009) believe that the FCN period is stable with no obvious evidence of time variation; however, some researchers (Xu and Sun 2009; Cui et al. 2014) disagree.

This problem remains a matter of dispute due to some deficiencies in the current understanding. The FCN period variation measured by VLBI is usually within one sidereal day (Vondrák and Ron 2009; Cui et al. 2014). With the use of effective angular momentum functions (EAMFs), the fluctuation in the FCN period becomes larger (within 3 sidereal days) instead of disappearing after considering the atmospheric and oceanic effect to forced nutation terms (Vondrák and Ron 2010; Cui et al. 2014), which means the fluctuation is not the result of geophysical fluids. Comparison with SG results is required to further verify the FCN temporal fluctuation. In previous research, the FCN period, obtained from a station in Strasbourg, shows similar temporal variations to the VLBI result (Cui et al. 2014). However, the FCN period fluctuation from SGs is up to several even dozens of sidereal days, which is much larger than the VLBI result; the fluctuations measured at different stations do not agree well with the Strasbourg station. The SG observations theoretically provide more opportunities to detect this variation due to the large number of stations in the global SG network. The global accumulation of gravity data offers many time series to compare the FCN period variation. If the FCN period does contain a temporal variation, all the SG sites around the world should observe a common variation.

In this paper, the gravity observation series were simulated by synthesizing the diurnal tidal waves, which are affected most by the FCN resonance, and adding white noise to adjust the estimated precision of tidal parameters ("Synthetic data" section). Then, the reason for the SG-observed FCN period fluctuation being much larger than that of VLBI was analyzed, and the FCN resonance variation detection condition in Earth tides, under the current precision of SG observations, was found ("The fluctuation of FCN period from SG observation" and "The detectability of FCN period change in diurnal tidal waves" sections). Based on the analysis with synthetic data, tidal analysis was completed with 20 long series from global SG stations to analyze the detectability of
FCN resonance variation in the current global SG observations and find the real fluctuation of the FCN period from SG observations ("Global SG data" section).

\section{Synthetic data}

In real SG observations, the true value of the FCN period is unknown. Thus, in order to answer the aforementioned questions, the best approach is to simulate gravity observations with synthetic data in which a known period is set. Observations with SG instruments include many signals, including the Earth's tidal waves, instrument drift, atmospheric and oceanic load effects, pole tide and noises. In tidal analysis, these terms, except noise, can be dealt with using the correct methodology. Since FCN only causes resonance enhancement in diurnal tidal waves with nearby frequencies, only the six tidal waves $(\mathrm{Q} 1, \mathrm{O} 1, \mathrm{P} 1, \mathrm{~K} 1, \psi 1$ and $\phi 1)$ are considered in the synthetic process for convenience. These tidal waves are usually used to retrieve the FCN parameters. The resonance formula between the FCN and tidal waves can be described as (Ducarme et al. 2007):

$$
\tilde{\delta}(\sigma)=\delta_{0}+\frac{\tilde{a}}{\sigma-\tilde{\sigma}_{\mathrm{FCN}}}
$$

Here, $\sigma$ is the frequency of a tidal wave; $\tilde{\delta}$ is the observed amplitude factor; $\delta_{0}$ is the amplitude factor, independent of the frequency and uninfluenced by the FCN resonance; $\tilde{a}$ is the complex resonance strength, related to the geometric parameters and Love numbers of the Earth; $\tilde{\sigma}_{\mathrm{FCN}}$ is the complex eigenfrequency of the FCN.

Based on Eq. (1), the complex eigenfrequency and complex resonance strength of FCN were set to calculate the theoretical amplitude factor, with which the synthetic data were constructed:

$$
y(t)=\delta_{i} A_{i} \cos \left(\omega_{i} t+\varphi_{i}+\Delta \varphi_{i}\right)+\varepsilon(t)
$$

where $y(t)$ is the synthetic gravity observation; $\delta_{i}$ and $\Delta \varphi_{i}$ are the amplitude factor and phase lag, respectively, for the $i$ th wave; $A_{i}, \omega_{i}$ and $\varphi_{i}$ are the theoretical amplitude, frequency and initial phase for the $i$ th wave, respectively; and $\varepsilon(t)$ is the white noise with which the precision of tidal parameters was simulated to be close to the level currently achieved in tidal analysis with actual superconducting gravimeter observations.

\section{The fluctuation of FCN period from SG observation}

The FCN parameters in previous works are usually determined by the least square method or Bayesian method. Based on Eq. (1), the merit function is usually constructed by using the $\mathrm{O} 1$ wave as a reference to reduce the effects of some systematic discrepancies. Subtracting the contribution of $\mathrm{O} 1$ from both sides of Eq. (1), the 
merit function $f$ can be written as (Defraigne et al. 1994; Sun et al. 2003):

$$
f=\sum_{\sigma} p(\sigma)\left|\left[\tilde{\delta}-\tilde{\delta}_{\mathrm{O} 1}\right]-\left[\frac{\tilde{a}}{\sigma-\tilde{\sigma}_{\mathrm{FCN}}}-\frac{\tilde{a}}{\sigma_{\mathrm{O} 1}-\tilde{\sigma}_{\mathrm{FCN}}}\right]\right|^{2}
$$

in which $p(\sigma)$ is the weight function of each tidal wave with the frequency $\sigma$. The weight function of each wave is related to the standard deviation of the tidal parameters $(\mathrm{d} \delta)$. In previous research, the most commonly used weight is inversely proportional to the standard deviation $(\mathrm{P} 1=1 / \mathrm{d} \delta)$ or to the square of it $\left(\mathrm{P} 2=1 / \mathrm{d} \delta^{2}\right)$. Another weight is added the frequency difference between a tidal wave $(\sigma)$ and $\mathrm{FCN}\left(\sigma_{\mathrm{FCN}}\right)$ to $\mathrm{P} 1\left(\mathrm{P} 3=1 / \mathrm{d} \delta\left(\sigma-\sigma_{\mathrm{FCN}}\right)\right)$. Since $\mathrm{d} \delta$ is inversely proportional to the amplitude of the wave, the weight of $\mathrm{K} 1$ is dominant over other diurnal waves and the weight of $\psi 1$ is very small.

In order to check the fluctuation of FCN period from SGs, a synthesized data series group was constructed in which a sinusoidal variation, with peak-to-trough amplitude of 4 SDs, was set for the FCN period according to Eq. (1). Then, the tidal parameters of waves in each data series were estimated by the least square method. Every synthetic time series is 1 year in length. By adding white noise, the standard deviations of the fitted tidal parameters were adjusted to replicate high-quality SG observations data. The FCN period was then estimated using the least square method and the weights $\left(\mathrm{P} 2=1 / \mathrm{d} \delta^{2}\right)$.

The FCN period and the amplitude factors of the K1 and $\psi 1$ waves are plotted in Fig. 1 . The sinusoidal waveform varies significantly in wave $\mathrm{K} 1$, but there is large deviation in the variation in wave $\psi 1$ and the fitted FCN period. The variation amplitude in the FCN period is over dozens of SDs, which is much larger than the set value of 4 SDs. According to correlation analysis, the correlation coefficient between the $\psi 1$ wave and the FCN period is as high as -0.98 , whereas the correlation coefficient between the $\mathrm{K} 1$ wave and the FCN period is only -0.55 . Therefore, the fitted period fluctuation is mainly due to the amplitude factor of the $\psi 1$ wave with a variation of 0.016 , which corresponds to a variation over $10 \mathrm{SDs}$ in the FCN period. The 4 SDs sinusoidal variation has been correctly reflected in the amplitude factor of the $\mathrm{K} 1$ wave with a variation of roughly 0.0002 . However, this is not shown in the FCN period result.

The correlation between the $\psi 1$ wave and the FCN period is the same in actual SG observations (Rosat et al. 2009). The variation in the FCN period depends on the $\psi 1$ wave's amplitude factor or its real part (since the phase difference is small, the two values are not significantly different) independent of the chosen numerical method (Cui et al. 2014). This is because the $\psi 1$ wave is closest to the FCN and affected most by FCN resonance.

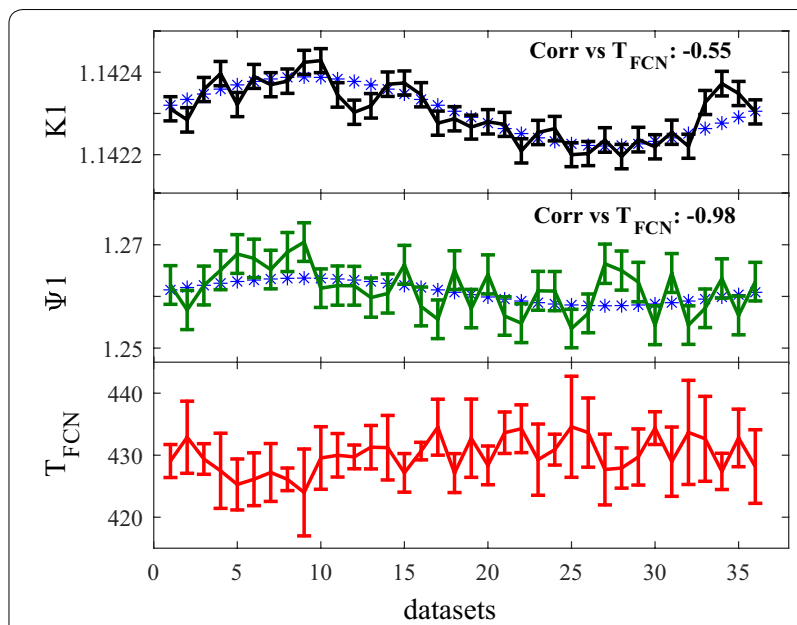

Fig. 1 Fluctuation of the FCN period (red) and the K1 (black) and $\psi 1$ (green) waves fitted from synthetic time series. The blue stars in top and middle panels are the theoretical values of amplitude factor of $\mathrm{K} 1$ and $\psi 1$ waves corresponding to the set FCN period in synthetic data. The FCN period (red) is estimated with the tidal parameters of 6 diurnal waves. The "Corr versus $T_{\text {FCN }}$ " represents the correlation coefficient between $\mathrm{K} 1$ and $\psi 1$ waves and $\mathrm{FCN}$ period

However, it has a relatively lower signal-to-noise ratio due to its small amplitude. In the calculation, the weight of $\psi 1$ is minute compared with the weight of the K1 wave, but still cannot reduce its impact. The large period fluctuation found in SG observations and the discrepancies observed among different stations could be caused by this limitation in the $\psi 1$ wave.

\section{The detectability of FCN period change in diurnal tidal waves}

In order to calculate the FCN period variation, it is necessary to find the detectability of the FCN period change in diurnal Earth tides under the current precision of SG observations. The detectability of the FCN resonance change depends on the precision of tidal parameters, the size of the FCN period change and the FCN resonance enhancement in tidal waves.

The precision of tidal parameters depends on the quality of SG observations, which relates to the station environment and instrument status. For a single tidal wave, the precision is inversely proportional to the wave's amplitude. The precision for actual SG observations varies significantly between stations. According to previous research (Cui et al. 2014), the standard deviation of the amplitude factor for diurnal waves can achieve Q1-2 $\times 10^{-4}, \mathrm{O} 1-4 \times 10^{-5}, \mathrm{P} 1-9 \times 10^{-5}$, $\mathrm{K} 1-3 \times 10^{-5}, \psi 1-4 \times 10^{-3}, \phi 1-2 \times 10^{-3}$, which could represent high precision data in the current global SG stations. This is also shown in the tidal analysis results 
for 20 global stations, which is analyzed in more detail in "Global SG data" section. For some stations, the standard deviation could be one or two orders of magnitude larger than above one.

Currently, the exact magnitude of the $\mathrm{FCN}$ period change is unknown. SG observation results are not suitable for reference due to the aforementioned issue. The FCN period determined from VLBI varies on the order of 3 SDs after correcting the geophysical effects with atmospheric and oceanic EAMFs. However, there are some problems with this correction, especially with the oceanic effect, as mentioned in "Introduction" section. Thus, in general, the magnitude of the FCN period change could be a few sidereal days, considering some uncertainties.

For quantitative analysis, the amplitude factors of diurnal tidal waves varying with the FCN period are estimated in Fig. 2, which clearly displays the ability of these diurnal waves to detect the variation in FCN resonance. The high precision level of tidal parameters mentioned before is denoted by the dash dotted line as a reference value. Only the $\mathrm{K} 1$ and $\psi 1$ waves exceeded the reference line and therefore have the potential to identify the changes in the FCN period in the diurnal band. The error in the tidal parameters of other waves is far larger than the variation caused by FCN period change. In addition, Fig. 2 indicates that $\mathrm{K} 1$ is more sensitive than $\psi 1$ in detecting the FCN period variation. The $\mathrm{K} 1$ amplitude variation can achieve observation precision when the FCN period varies by more than 1

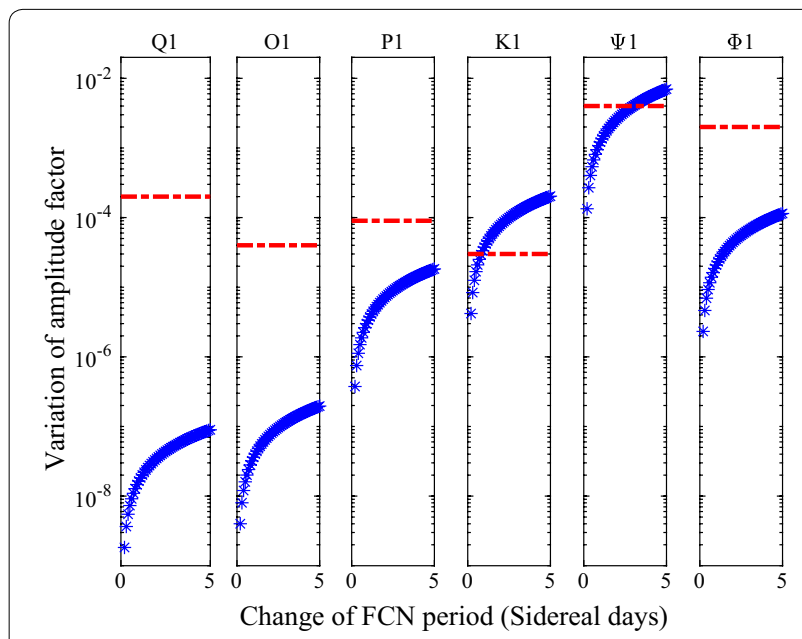

Fig. 2 Relationship between the variations in amplitude factor and the FCN period. The horizontal axis represents the change of FCN period. The vertical axis is the corresponding variation in amplitude factors for 6 diurnal waves. The dash dotted line represents the high precision level of tidal parameters from the current global SG observations
SD. For the $\psi 1$ wave, observation precision is possible until the FCN period varies by more than about 2.5 SDs.

Considering the FCN period change magnitude and the current precision of tidal parameters, only the observations with the highest precision level can detect FCN period variation in the $\psi 1$ wave. By setting the amplitude of the sinusoidal variation similarly to Fig. 1, the $\psi 1$ amplitude factor variation was estimated (Fig. 3a). The sinusoidal temporal variation in $\psi 1$ could be observed, along with the increase in period variation. However, even if the period variation increases to $5 \mathrm{SDs}$, some large deviations remain.

For the $\mathrm{K} 1$ wave, the variation for a few days of FCN period change under a high precision level can be observed (Fig. 1). It is necessary to find the accuracy required to clearly detect the variation. Data series with different STD_K1 (the standard deviation of the K1 amplitude factor) levels were constructed by adjusting the added white noise and setting a sinusoidal variation with an amplitude of 2 SDs (wave peak-to-trough change was 4 SDs) in synthetic datasets. The fitted K1 amplitude factors are listed in Fig. 3b. The sinusoidal trend was obtained correctly in the high precision datasets where
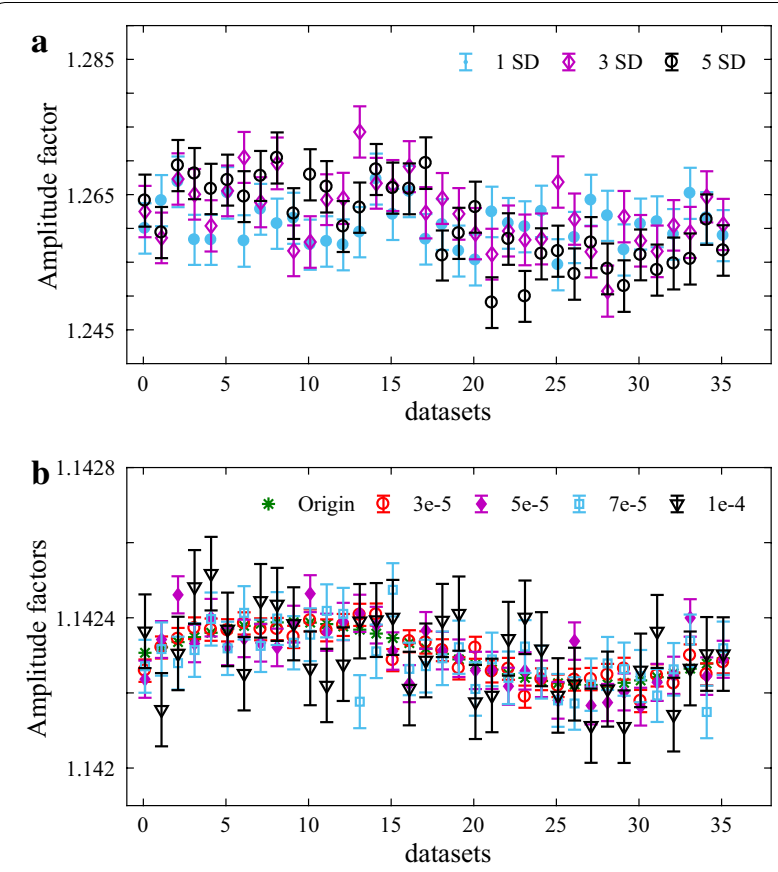

Fig. 3 a Sensitivity of $\psi 1$ to FCN period variation. Three different markers represent the variation in amplitude factor for $\psi 1$ wave caused by different FCN period changes in 36 synthetic time series; b sensitivity of $\mathrm{K} 1$ to $\mathrm{FCN}$ period variation. The green star is the theoretical FCN period set in 36 synthetic time series. The other four markers represent the variation in amplitude factor for $\mathrm{K} 1$ wave under different observation precisions 
STD_K1 is below $5 \times 10^{-5}$. When STD_K1 exceeded $5 \times 10^{-5}$ to $7 \times 10^{-5}$ or $1 \times 10^{-4}$ (Fig. 3b), the fitted results deviated from the true value and produced a false trend.

Overall, in diurnal tidal waves, only $\mathrm{K} 1$ and $\psi 1$ can detect FCN resonance change. The accuracy of the $\psi 1$ wave is relatively lower; thus, the FCN period variation obtained with SG observations deviates significantly due to its correlation with the $\psi 1$ wave. The $\mathrm{K} 1$ wave is more accurate than the $\psi 1$ wave and has good detectability when the STD_K1 is below $5 \times 10^{-5}$.

\section{Global SG data}

Based on the synthetic test data, the following research was performed based on the time series of gravity observation recorded at 20 SG stations in the IGETS (International Geodynamics and Earth Tide Service) network, the previous Global Geodynamics project (GGP). The GGP was organized by the Solid Earth's Deep Interior (SEDI) in IUGG (The International Union of Geodesy and Geophysics) in 1997. Since 2016, a new IAG (the International Association of Geodesy) service, called IGETS, continues the activities of the GGP to provide support to the research activities using SG data within the context of an international network. The partners in IGETS use uniform data collection equipment, central sensors and a low-pass filter (Crossley and Hinderer 1995). All the datasets were preprocessed with the T-soft software (Van Camp and Vauterin 2005) to deal with bad records, such as gaps, spikes, steps and Earthquakes, before carrying out the tidal analysis. The datasets used in this study were all sampled hourly and mostly accumulated over 10 years. The basic information, e.g., station names, instruments, observing periods and station location, is listed in Table 1. In Table 1, note that stations with "*;" are closed, and that $(\mathrm{L} / \mathrm{U})$ represents the double sphere type in which only one sphere's observation was chosen because the tidal parameters obtained from the double sphere observations were in close agreement.

\section{Atmospheric and ocean tide loading effects}

As the most important noise source in tidal gravity observations, it is necessary to effectively eliminate the influence of atmospheric pressure and ocean tide loading in order to improve the accuracy of the tidal parameters (Boy et al. 2002; Baker and Bos 2003). By using the atmospheric pressures observed at each SG station with high sampling, the effect of atmospheric pressure is eliminated simultaneously in the tidal analysis according to

Table 1 SG stations and observation information

\begin{tabular}{|c|c|c|c|c|c|c|}
\hline \multirow[t]{2}{*}{ No } & \multirow[t]{2}{*}{ Station name } & \multirow[t]{2}{*}{ SG type } & \multirow{2}{*}{$\begin{array}{l}\text { Observation period } \\
(\mathrm{Y} / \mathrm{M}-\mathrm{Y} / \mathrm{M})\end{array}$} & \multicolumn{3}{|c|}{ Station location } \\
\hline & & & & Longitude $\left({ }^{\circ}\right)$ & Latitude $\left({ }^{\circ}\right)$ & Height(m) \\
\hline 1 & BE: Brussels, Belgium & *T003 TT70 & 1982-04-2000-09 & 4.358 & 50.7986 & 100.0 \\
\hline \multirow[t]{2}{*}{2} & BH: Bad Homburg, Germany & CD030 (L/U) & 2001-02-2007-04 & 8.6113 & 50.2285 & 190.0 \\
\hline & & SG044 & 2007-02-2013-02 & & & \\
\hline \multirow[t]{2}{*}{3} & CA: Cantley, Canada & T012 TT70 & 1989-11-1993-10 & 284.1927 & 45.5850 & 269.2 \\
\hline & & & 1997-07-2015-03 & & & \\
\hline 4 & CB: Canberra, Australia & C031 & $1997-07-2014-12$ & 149.0077 & -35.3206 & 762.7 \\
\hline 5 & CO: Conrad,Austria & $\mathrm{CO25}$ & 2007-11-2013-11 & 15.8609 & 47.9288 & 1045.1 \\
\hline 6 & KA: Kamioka, Japan & T016 & 2004-10-2013-07 & 137.3084 & 36.4253 & 358.0 \\
\hline 7 & MA: Matsushiro, Japan & T011 TT70 & 1997-05-2006-06 & 138.2302 & 36.5439 & 409.5 \\
\hline 8 & MB: Membach, Belgium & $\mathrm{C} 021$ & 1995-08-2011-12 & 6.0066 & 50.6093 & 250.0 \\
\hline 9 & MC: Medicina, Italy & $\mathrm{CO23}$ & $1998-01-2013-12$ & 11.645 & 44.5219 & 28.0 \\
\hline 10 & ME: Metsahovi, Finland & T020 TT070 & 1997-07-2012-07 & 24.3958 & 60.2172 & 55.6 \\
\hline 11 & MO: Moxa, Germany & CD034 (L/U) & 2000-01-2013-07 & 11.6156 & 50.6447 & 455.0 \\
\hline 12 & NY: Ny-Alesund, Norway & C039 & 1999-09-2011-12 & 11.8672 & 78.9306 & 43.0 \\
\hline 13 & PE: Pecny, Czech Republic & OSG-050 & 2007-05-2014-12 & 14.7856 & 49.9138 & 534.6 \\
\hline 14 & ST: Strasbourg, France & C026 & 1997-03-2011-12 & 7.6850 & 48.6217 & 185.0 \\
\hline 15 & SU: Sutherland, South Africa & D037 (L/U) & 2000-03-2014-12 & 20.8109 & -32.3814 & 1791.0 \\
\hline 16 & SY: Syowa Antarctica & T016 & 1997-07-2003-01 & 39.5857 & -69.0067 & 42.6 \\
\hline 17 & TC: TIGO Concepcion, Chile & T038 & $2002-12-2014-12$ & 291.9745 & -36.8437 & 100.0 \\
\hline 18 & Vl: Vienna, Austria & ${ }^{*} \mathrm{C} 025$ & 1997-07-2006-12 & 16.3579 & 48.2493 & 192.4 \\
\hline 19 & WE: Wettzell, Germany & CD029 (L/U) & 1998-11-2010-10 & 12.8780 & 49.1440 & 613.7 \\
\hline 20 & WH: Wuhan, China & T004 TT70 & 1998-01-2010-12 & 114.4898 & 30.5159 & 80.0 \\
\hline
\end{tabular}


the response functions of gravity to air pressure in the tidal band (i.e., the atmospheric gravity admittances), which is evaluated by regression analysis (Merriam 1992).

Since oceanic tides have the same forces and a similar spectral pattern to body tides, harmonic analysis cannot separate them. Therefore, the ocean tide loading effect is usually corrected using an ocean tide model. In this study, the three newest high-resolution models were chosen: FES2012 (Carrère et al. 2012), FES2014 (Carrère et al. 2015) and TPXO8-Atlas (Egbert and Erofeeva 2002), with which the ocean loading effect is calculated from the Free Ocean Tide Loading Provider (Bos and Scherneck 2014). In the diurnal band, the ocean tide models provide only the cotidal maps of four waves (Q1, $\mathrm{O} 1, \mathrm{P} 1$ and $\mathrm{K} 1$ ); the loading effects for the major waves of tidal gravity are shown in Fig. 4. The loading effects of the $\psi 1$ and $\phi 1$ waves are usually interpolated using linear regression from the major waves (Xu et al. 2004).

The loading effects of ocean tides on the $\mathrm{O} 1$ and $\mathrm{K} 1$ waves are generally larger than those on the P1 and Q1 waves as the effects are proportional to the amplitude of these waves. Remarkably, the loading effects, globally, depend on the distance between the station and the sea or the strength of the oceanic tide around the station. At coastal stations such as Canberra, Kamioka, Matsushiro, TIGO Concepcion and Ny-Alesund, the loading effects are significantly stronger. The results from the offshore Syowa station are extremely large, and possibly inaccurate, as the three newest ocean tide models in this paper offer far larger values than previous models, such as Fes2004 (Table 2, in Sun et al. 2009). In contrast, inland stations, especially those located in Europe, have much lower loading effects in the diurnal band due to the small adjacent diurnal ocean tides. However, the M2 wave is very different. The loading effects of the M2 wave in these European stations are about 2 orders of magnitude larger than those for the $\mathrm{K} 1$ or O1 waves, whose amplitude are similar to the M2 wave. This is important as the modulation of M2 in some European stations is very obvious (Meurers et al. 2016), but diurnal wave modulation is weak.

It is worth noting that the present ocean tide model cannot provide time-varying ocean loading effects, which is a possible source of temporal variation in the tidal parameters (Calvo et al. 2014). This study focuses on the FCN resonance variations in diurnal tidal waves, which primarily affect the $\mathrm{K} 1$ and $\psi 1$ waves adjacent to the FCN frequency, as shown in Fig. 2. Time variance in the ocean

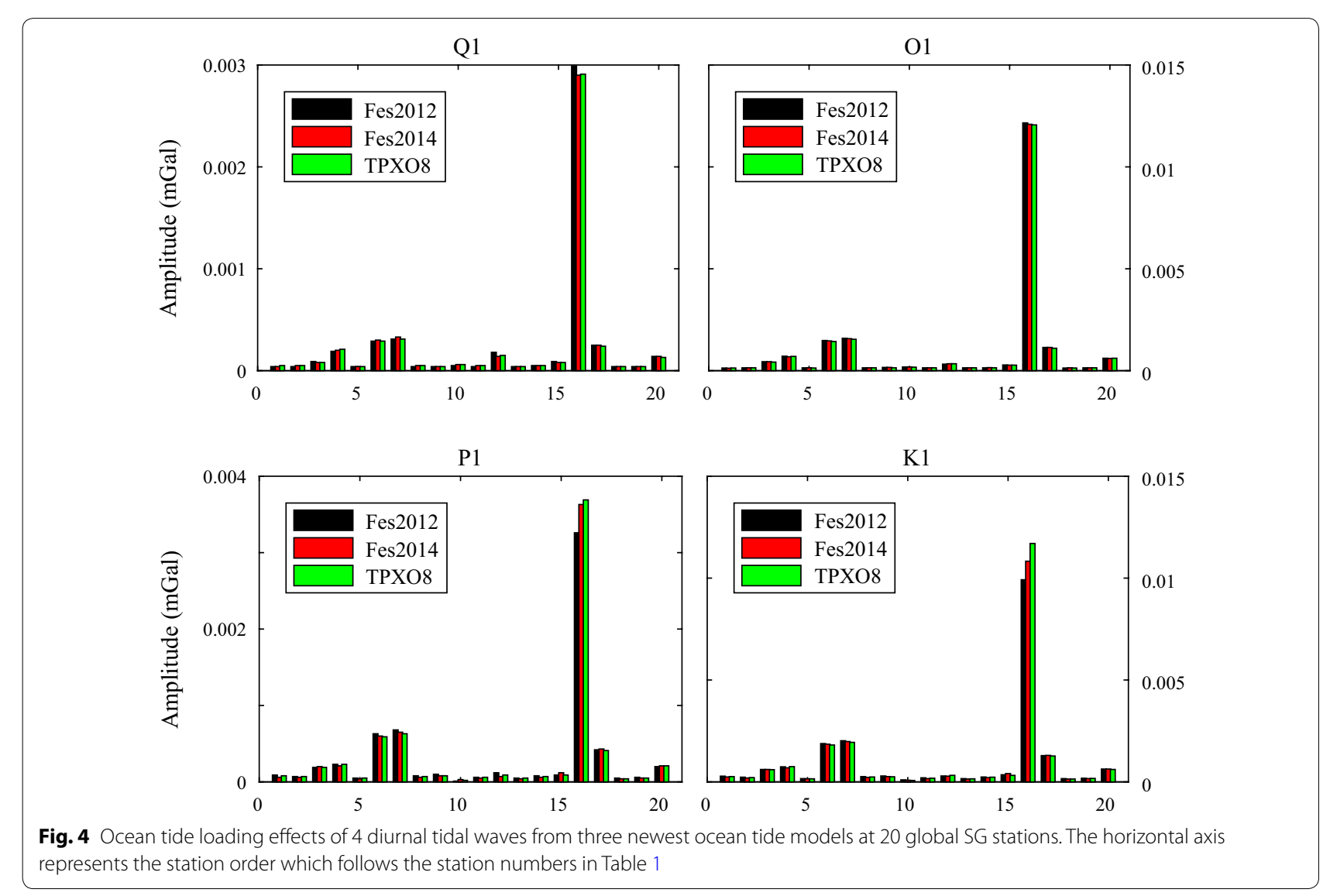


loading effect should appear in all diurnal tidal waves of one station with a similar pattern. Thus, by comparing the variation in diurnal waves and at stations located in different regions, the resonance variation can be distinguished from the time-varying ocean loading effect. Moreover, for most stations, SG instruments are installed in quiet inland areas, far from the ocean where the ocean tide effect is small.

\section{Tidal analysis}

Using the tidal analysis software ETERNA (Wenzel 1996), the tidal parameters of selected wave groups were estimated from the 20 observations in Table 1, based on the Hartmann and Wenzel (1995) tidal potential catalog. A 3-year data window was chosen to separate the diurnal tidal waves focused on in this study. To obtain the tidal parameter variation in tidal waves, the 3-year data window, with a 1-year overlap, was shifted for each observation. The results indicate that the estimated tidal parameters of the diurnal tidal waves, from 20 observations, vary too much from station to station. In Fig. 5, the real part of the amplitude factors of the K1 (left) and $\psi 1$ waves (right) in 20 stations is given. The results of the K1 wave at the SY station, listed separately, are appreciably different from other stations because the ocean tide loading effects are too large and cause inaccuracies at this station, as mentioned above.

From the tidal analysis results of the $\mathrm{K} 1$ and $\psi 1$ waves, it can be seen that the precision of tidal parameters across global stations greatly varies, which is related to the observation quality at these stations. Therefore, the tidal parameters of a few stations vary much more than others. As indicated in Calvo et al. (2014), the local noise variation could be a major cause of observed fluctuations in tidal parameters. In addition, there are other possible errors in the amplitude factors, such as scale problems at some stations like TC. Moreover, certain instrument problems can cause similar errors. Therefore, it is difficult to gather effective information just from the results in Fig. 5.

As discussed in "Synthetic data" section, it is necessary to include the observation precision of each diurnal wave in order to obtain reliable analyses. In Fig. 6, the standard deviation of the K1 wave amplitude factor for each segment of the 20 stations is listed. The precision level of

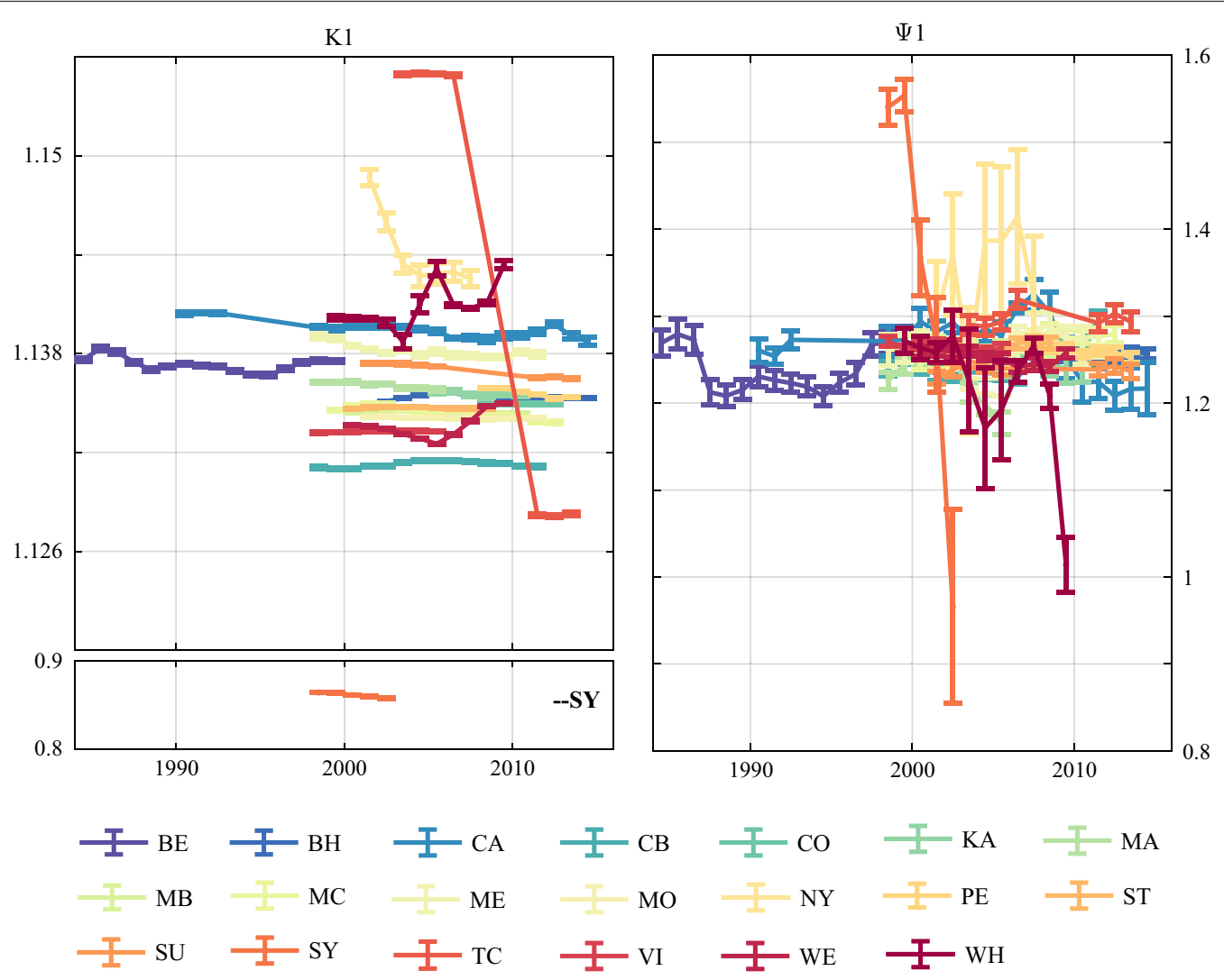

Fig. 5 Tidal analysis results of 20 observations from global stations in Table 1, in which the variation in the amplitude factor (real part) of the K1 (left) and $\psi 1$ (right) waves is plotted with error bar. The abbreviation of station name in the legend follows Table 1 

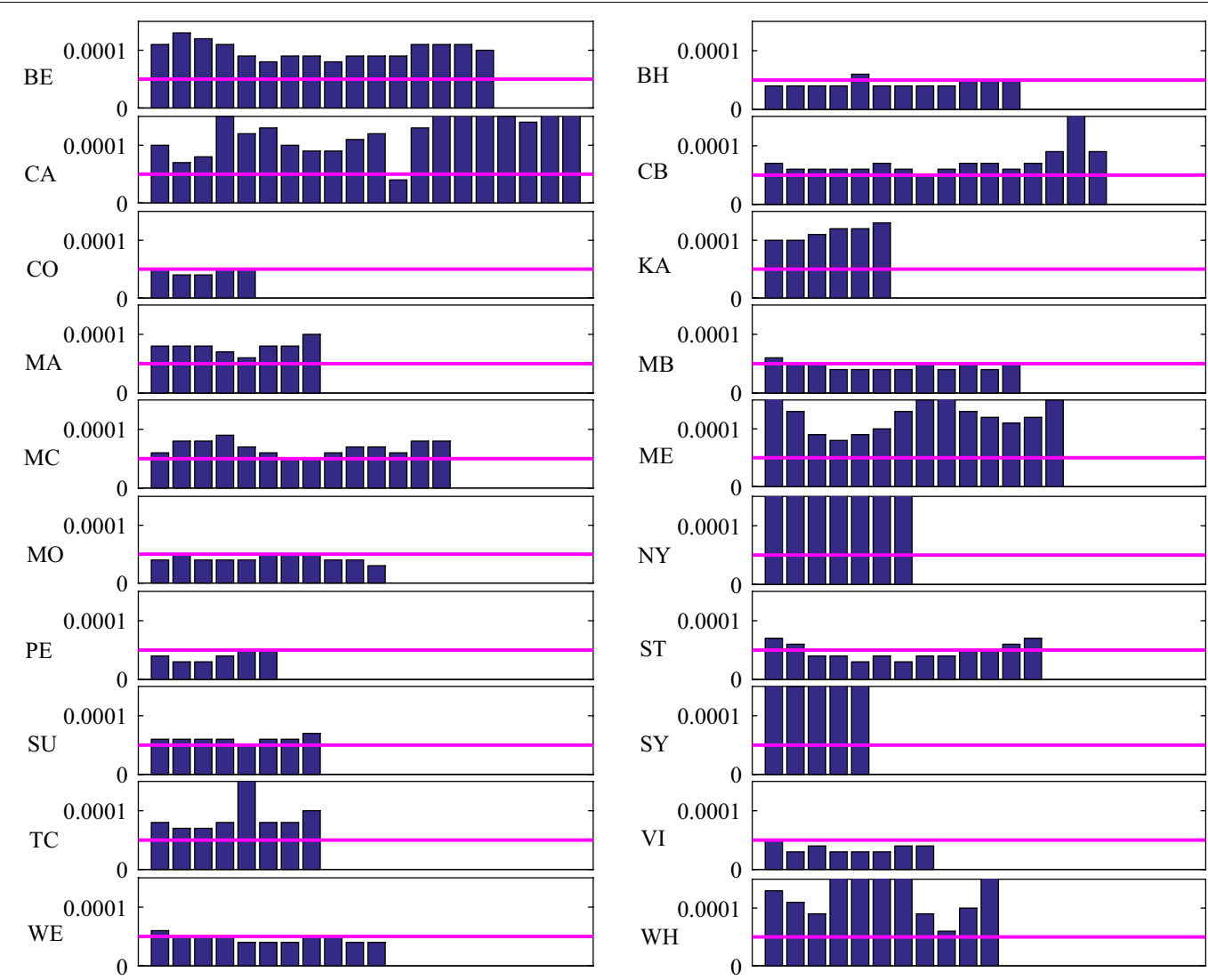

Fig. 6 Tidal analysis precision of 20 global stations. The vertical axis is the standard deviation of the amplitude factor of the K1 wave. The horizontal axis is the number of data segments by sliding time window to each observation. The violet line is the reference line for a standard deviation of $5 \times 10^{-5}$

other waves is consistent with the K1 wave in the comparison between different stations, because if the station has little background noise and high data quality, the standard deviation for each wave is, overall, relatively small, and vice versa. From Fig. 6, most European stations, except $\mathrm{BE}, \mathrm{MC}$ and $\mathrm{NY}$, have high precision observations when STD_K1 is in the range of $3-5 \times 10^{-5}$. The observations from other stations have larger STD_K1 levels due to relatively lower data quality. Few stations have very low precision, due to the station location, e.g., high latitude stations (SY and NY) where the amplitude of diurnal tidal waves is very small. The gaps in continuous observations, due to electric power or instrument failure, also significantly affect the standard deviation of tidal parameters. This is inevitable for every station; sometimes, the effect is very large, such as in the fifth segment of the TC station.

Observations with the standard deviation below $5 \times 10^{-5}$ have a good detectability to the variation in the FCN period for the K1 wave ("Synthetic data" section). As the standard deviation increases, large discrepancies can be observed. Thus, in order to effectively analyze the results from global SG observations, the 20 stations in Table 1 were divided into three classes. The first class includes station where the STD_K1 is below $5 \times 10^{-5}$. Stations with an STD_K1 in the range of $5-10 \times 10^{-5}$ belong to the second class. The remaining stations, with an STD_K1 greater than $1 \times 10^{-4}$, are the third class. The amplitude factors of the diurnal waves (O1, P1, K1 and $\psi 1$ ) for the three station classes are given in Figs. 7, 8 and 9. In Figs. 7 and 8, which show the first and second station classes, respectively, the few segments of some stations, with an STD_K1 outside the classification range, are excluded.

In order to avoid errors in the scale factor and changes in the sensitivity of the gravimeter, it is very effective to use the $\mathrm{O} 1$ wave as a reference (e.g., Ducarme et al. 2007) due to its relatively large amplitude, high signal-to-noise ratio and resistance to the FCN resonance. Therefore, in Figs. 7, 8 and 9, the amplitude factor of the $\mathrm{O} 1$ wave was used to normalize the P1, K1 and $\psi 1$ waves. For the convenience of comparison, the normalized results (including $\mathrm{O} 1$ wave) were then divided by their mean value in each timespan. 


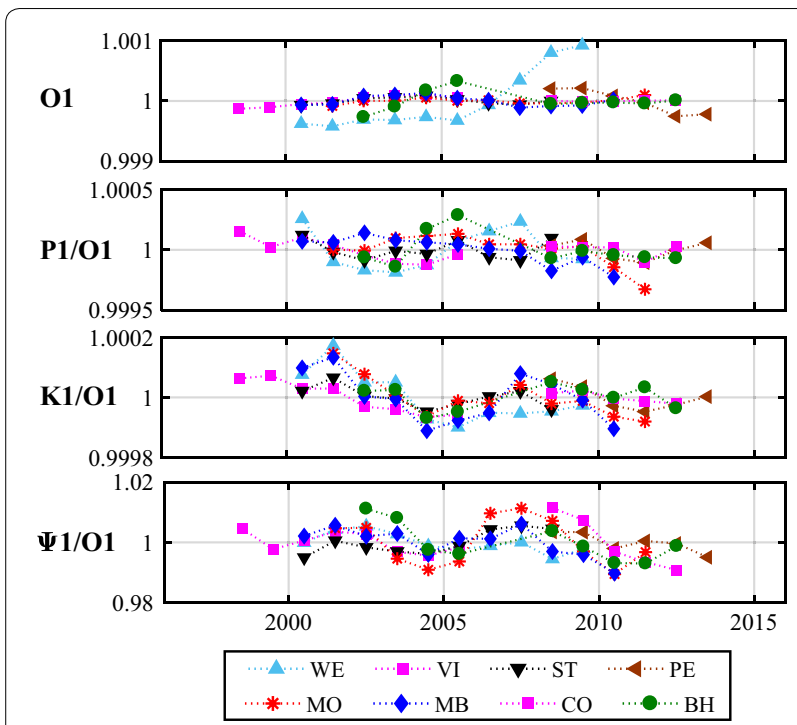

Fig. 7 Amplitude factor of diurnal waves in the first station class. Amplitude factors of $\mathrm{P} 1, \mathrm{~K} 1$ and $\psi 1$ waves are normalized by the one of the $\mathrm{O} 1$ waves. All amplitude factors are then divided by their mean value for the convenience of comparison

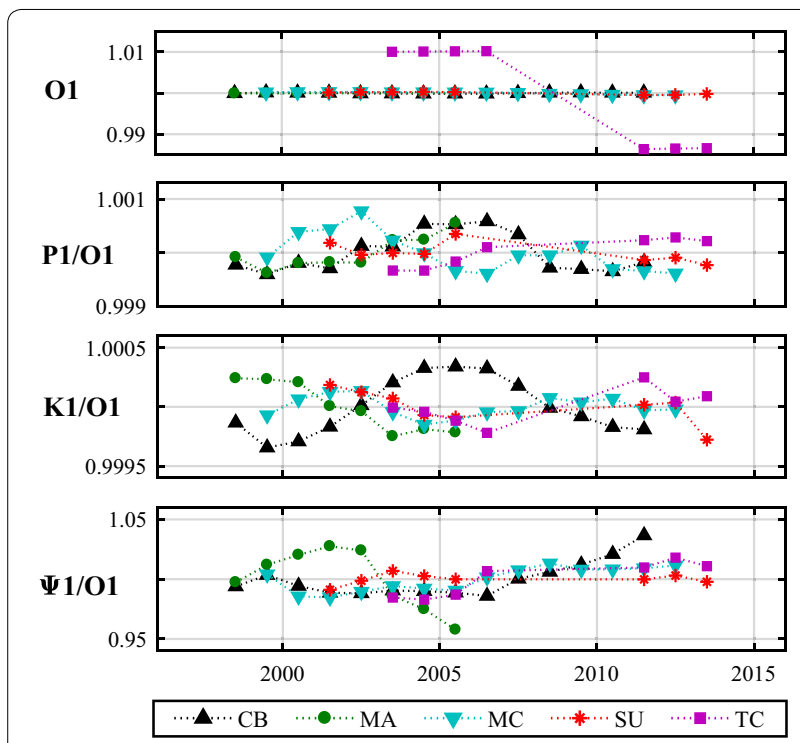

Fig. 8 Amplitude factor of diurnal waves in the second station class. Amplitude factors of $\mathrm{P} 1, \mathrm{~K} 1$ and $\psi 1$ waves are normalized by the one of the $\mathrm{O} 1$ waves. All amplitude factors are then divided by their mean value for the convenience of comparison

As the reference wave, no effective information can be used from the variation in the $\mathrm{O} 1$ wave. Remarkably, from the other 3 waves, a consistent trend occurs in the amplitude factor of the $\mathrm{K} 1$ wave for all 8 stations in Fig. 7. The temporal variations in the 8 first station class cover roughly the same time period, over 10 years. Stations CO and VI can be considered as one station as they use the same SG instrument (GWR C025), which was transferred in the autumn of 2007 from VI to CO (60 km southwest of VI) after 12 years' observation (Meurers 2012). Furthermore, the amplitude factor variations in the $\psi 1$ wave at these stations are self-consistent and similar to the $\mathrm{K} 1$ wave. Only a few points at the WE station deviate from the trend during the period between 2006 and 2008. Figure 7 shows that the amplitude factor of $\mathrm{O} 1$ wave has obvious larger variation, possibly due to certain errors such as scale factor problem. The variations in the K1 wave are more centralized, and the results of the $\psi 1$ wave are relatively discrete. If this variation is related to the FCN resonance source, this is because the $\mathrm{K} 1$ wave is more accurate in detecting the resonance variation caused by FCN period change, and the low precision of the $\psi 1$ wave results in increased deviation to the results. The P1 wave is also a large amplitude tidal wave (1/3 times that of $K 1$ and several dozen times that of $\psi 1$ ) and can be determined with high precision. However, in Fig. 7, there is no consistent trend like the $\mathrm{K} 1$ or $\psi 1$ waves in the variations in the P1 waves. This indicates that the temporal variation found in the $\mathrm{K} 1$ and $\psi 1$ wave is not a common effect for all tidal waves.

In addition, the stations (WE, $\mathrm{VI}+\mathrm{CO}, \mathrm{ST}, \mathrm{MO}$ ) in Fig. 7, except WE, BH and PE, have a high correlation in the temporal variation in the $\mathrm{O} 1$ gravimetric factors which may increase correlation of the K1/O1 and Psi1/ $\mathrm{O} 1$ ratios. Actually, some stations (ST, MB, MO, VI) also show consistent temporal variation for $\mathrm{K} 1$ wave and $\psi 1$ waves before normalization. In our previous researches (Cui et al. 2014), the $\psi 1$ factors of ST, MB, MO stations also have obvious correlation without normalization. What is more, if the normalization plays dominant role, we should also find high correlation in the temporal variation in P1/O1 factors, but there is not. Therefore, the consistency between the neighboring $\mathrm{K} 1$ and $\psi 1$ waves should not be mainly due to the normalization by $\mathrm{O} 1$.

In the second station class (Fig. 8), the MC station has similar amplitude factor variation trend for the K1 wave to the results of the first station class. In SU and TC stations, the observations between 2006 and 2012 were missed, but the variations in K1 before 2006 are consistent with the MC station. Moreover, the K1 variation magnitude of these 3 stations is larger than that of the first station class. It is notable that the consistent trend is not observed in the $\psi 1$ results in Fig. 8, which is attributed to the lower detectability of the $\psi 1$ wave FCN resonance change because of its larger standard deviation. The K1 wave result at the CB station has a completely different variation, which also is found in the amplitude factor of the P1 wave. Thus, this variation is not related to the FCN resonance variation, but is likely the temporal variation 

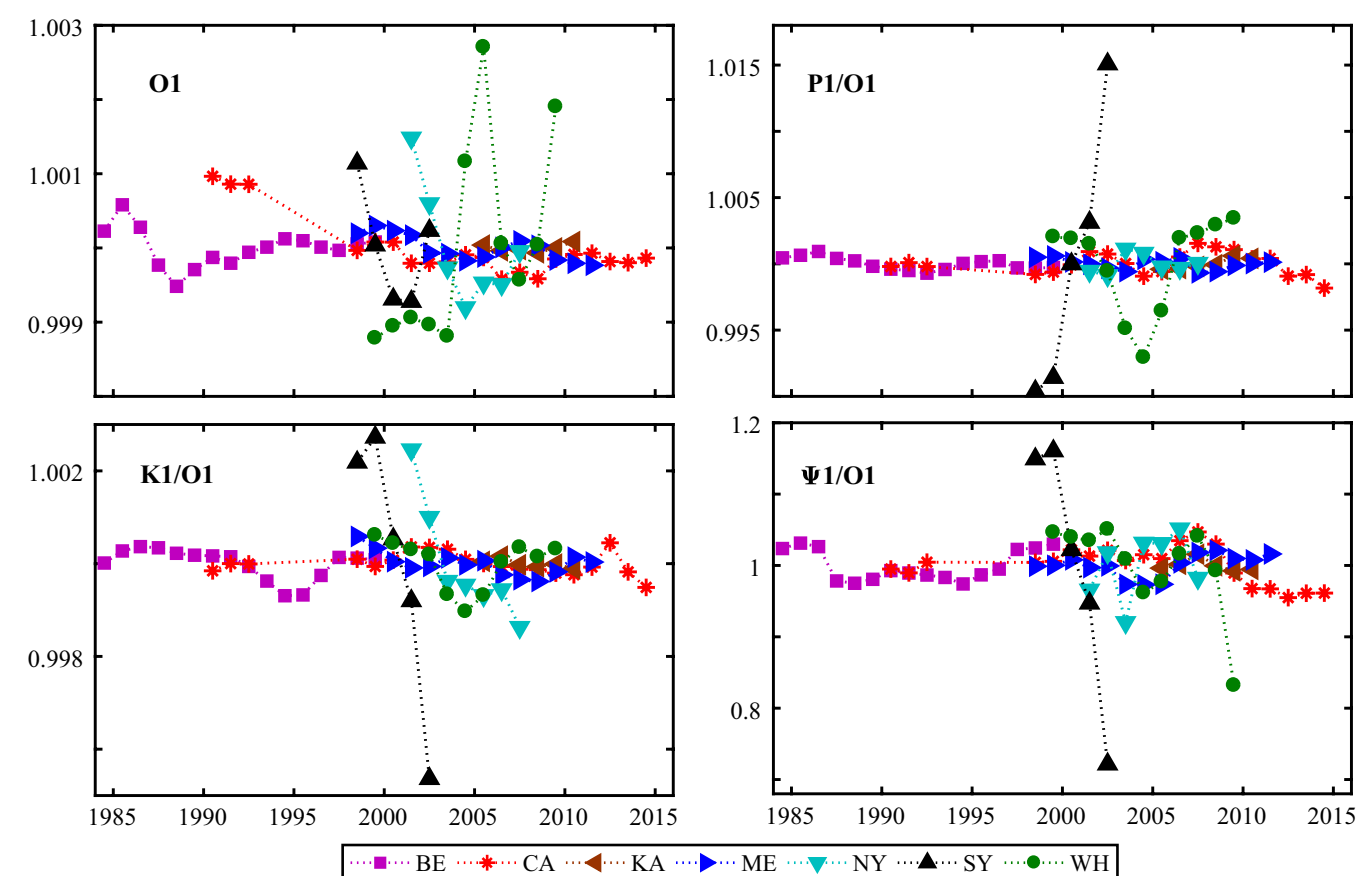

Fig. 9 Amplitude factor of diurnal waves in the third station class. Amplitude factors of $\mathrm{P} 1, \mathrm{~K} 1$ and $\psi 1$ waves are normalized by the one of the $\mathrm{O} 1$ waves. All amplitude factors are then divided by their mean value for the convenience of comparison

in the ocean tide load effect, because the CB station is very close to the sea and the ocean tide loading effect is larger at this station (Fig. 4), especially for larger waves, e.g., P1 and K1.

The third station class observations, which include 7 stations with an STD_K1 greater than $1 \times 10^{-4}$, cannot detect FCN resonance. Therefore, no effective information can be found in the amplitude factors from these stations.

\section{Discussion}

With the help of classification, it is found that a temporal variation occurs in the $K 1$ and $\psi 1$ waves of the first station class and in the $\mathrm{K} 1$ wave of some second-class stations. It is worth noting that there are many causes which could lead to temporal variations in the determined tidal parameters from SG observations, such as instrument calibration, preprocessing problems, numerical artefacts due to insufficient frequency, temporal variation in the load effect, Earth's structure or geodynamic processes (Meurers et al. 2016).

Firstly, instrument calibration and preprocessing problems can be excluded because these influences affect stations on an individual basis and cannot produce a common change in several stations. The temporal variations found in this study are also unlikely to be related to the Earth's structure or geodynamic processes which, if present, would be a large signal and not just affect the $\mathrm{K} 1$ and $\psi 1$ waves.

There is some seasonal effect not corrected from the influence of atmospheric pressure, which might influence the tidal parameter determination, particularly if short intervals (less than a year) are analyzed. This study focuses on long-term variation by using 3 years' worth of data. The temporal variation in ocean load is also an important cause for the changes in tidal parameters and should have a similar pattern in all diurnal waves of one station, but the common variation is only found in the $\mathrm{K} 1$ and $\psi 1$ waves and does not occur in another diurnal wave. Furthermore, the loading effect of ocean tides is very weak in the diurnal band of these high precision stations (Fig. 4). Therefore, this temporal variation is not due to ocean tide and atmospheric pressure loading.

The numerical artefacts due to an insufficient frequency, as detailed analyzed by Meurers et al. (2016), can also cause the temporal variations in the tidal parameters of the M2 wave. It is an important effect we cannot exclude now. But the numerical artefacts should affect all waves. The amplitude of P1 is much larger than $\psi 1$ and could be observed more precisely, but there is high correlation in the temporal variation in $\psi 1$ wave but not in P1 wave, which means the frequency resolution artifact may not be the main reason for this temporal variation. Moreover, the first station class (Fig. 7) are all European stations where 
the modulation of diurnal waves is relatively weak as mentioned in "Atmospheric and ocean tide loading effects" section. Therefore, the consistency between the neighboring $\mathrm{K} 1$ and $\psi 1$ waves suggests that this temporal variation is possibly due to $\mathrm{FCN}$ period variation.

What's more, comparing with previous researches, the temporal variation found in first station class (including Strasbourg station) further verifies the FCN period variation obtained from Strasbourg station in Cui et al. (2014), which agrees well with the fluctuation observed in VLBI results. From another point of view, the variation range of $\mathrm{K} 1 / \mathrm{O} 1$ in Fig. 7 is up to a value of about 0.0002 . This variation corresponds to a change of about 4 SDs in the FCN period. The variation in $\psi 1 / \mathrm{O} 1$ is about 0.02 , which corresponds to a change of more than 10 SDs in the FCN period. As analyzed in "Synthetic data" section, the variation in $\mathrm{K} 1$ reflects more exactly the FCN resonance variation. Furthermore, if the FCN resonance variation can be detected in both the $K 1$ and $\psi 1$ waves, the magnitude of the FCN period change should be greater than 2.5 SDs. Therefore, the variation observed in the $\mathrm{K} 1$ and $\psi 1$ waves provides a possible range of 2.5-4 SDs for the FCN period change. This is consistent with the FCN period results from VLBI observations.

In order to obtain a better understanding, it is needed to consider comprehensively above-mentioned factors and further compare with VLBI results. More importantly, the mechanism of the possible change in FCN period should be investigated. The FCN is caused by the interaction between the elliptical liquid core and the solid mantle. Its period mainly depends on these parameters related to the characteristics near CMB (Dehant et al. 2017), in which the dynamical flattening of outer core accounts for dominant proportion and other core mantle couplings (such as viscous, electromagnetic and topographic couplings) also play important role (Hinderer et al. 1982). The structural or physical parameters (such as dynamical flattening, viscosity, conductivity, topography) near core mantle boundary are supposed to be stable in the time scale of our study, although they will change with the evolution of the Earth in a long geological age. Thus, the most possible changes come from geomagnetic field changes sourced from large-scale hydrodynamic processes in liquid outer core. In Cui et al. (2018), we have estimated the effect of different core mantle couplings on FCN period. The influence of electromagnetic coupling can reach the magnitude of several sidereal days, which satisfy the possible FCN period changes in actual observations.

Furthermore, some recent researches (Malkin 2013; Vondrák and Ron 2015) indicate a possible correlation between FCN and geomagnetic jerk. The geomagnetic jerk is a relatively sudden change in the second derivative of the Earth's magnetic field with respect to time (De Michelis et al. 2005). The precise cause of geomagnetic jerk is still a matter of research but is more inclined to originating in the interior of the Earth rather than the external phenomena (Olsen and Mandea 2007). If geomagnetic jerk has correlation with FCN, the most possible mechanism is that geomagnetic jerk gives rise to the change of electromagnetic coupling at $\mathrm{CMB}$ which will contribute to the variation in the frequency or amplitude of FCN.

\section{Conclusions}

In this paper, the detectability of FCN period variation in Earth tides at the current precision level of SG instruments was evaluated by synthesizing time series. The results indicate that:

1. In FCN determination, the large discrepancy in the FCN period determined by SG observations is caused by the inaccuracy of the $\psi 1$ wave and the high correlation between the $\psi 1$ wave and the FCN period. The current approaches, such as the least square or Bayesian method, cannot overcome this defect. A new approach is needed for FCN parameter estimation using SG observations.

2. The $\mathrm{K} 1$ wave is the most sensitive to changes in the FCN period, and the corresponding resonance variation in $\mathrm{K} 1$ is more precise than that in the $\psi 1$ wave, despite the $\psi 1$ wave being closest to FCN and most affected by the resonance.

Based on the test with synthetic data, 20 long-term SG observations from global stations were analyzed. By classifying these stations according to their STD_K1, it was concluded that:

1. A consistent variation is found in both the $\mathrm{K} 1$ and $\psi 1$ waves in all 8 first-class stations. In the second station class, a similar fluctuation occurs in the K1 wave at some stations. The $\mathrm{K} 1$ and $\psi 1$ wave fluctuation constrains the $\mathrm{FCN}$ period change to the range of 2.5-4 SDs. Both the variation and the magnitude agree with the results from VLBI observations. These indicate the $\mathrm{FCN}$ period may be not so stable in time as it is generally believed.

2. According to the synthetic test and global SG observation, the temporal variation in the FCN period could be clearly detected when the STD_K1 from tidal analysis is below $5 \times 10^{-5}$.

3. Most of the common variations in Figs. 7 and 8 come from Europe. High-quality SG observations in other areas are required in future study. 


\section{Authors' contributions}

CUI participated in the design of the study, carried out the synthetic data experiment and analysis of superconducting gravimeter observations and drafted the manuscript. SUN and XU participated in the design of the study, carried out data analysis method and revised the manuscript. ZHOU carried out the correction of ocean tide loading effect. CHEN carried out the preprocess of superconducting gravimeter data. All authors read and approved the final manuscript.

\section{Author details}

1 State Key Laboratory of Geodesy and Earth's Dynamics, Institute of Geodesy and Geophysics, Chinese Academy of Sciences, Wuhan 430077, China. ${ }^{2}$ University of Chinese Academy of Sciences, Beijing 100049, China.

\section{Acknowledgements}

The authors are very thankful to the IGETS (the International Geodynamics and Earth Tide Service) database at GFZ Potsdam for providing global superconducting gravimeter observations. Also the authors are very thankful to Prof. Rosat and two anonymous reviewers for their remarks and suggestions which were helpful in preparing the revised version.

\section{Competing interests}

None of the authors have any competing interests in the manuscript.

\section{Availability of data and materials}

Please contact author for data requests.

\section{Consent for publication}

Not applicable.

\section{Ethics approval and consent to participate}

Not applicable.

\section{Funding}

This work was supported by the National Basic Research Program of China (973 Program) (2014CB845902) and National Natural Science Foundation of China $(41621091,41321063,41274085,41304058,41774092,41374084$ and 41574072).

\section{Publisher's Note}

Springer Nature remains neutral with regard to jurisdictional claims in published maps and institutional affiliations.

Received: 30 August 2018 Accepted: 7 December 2018

Published online: 18 December 2018

\section{References}

Baker TF, Bos MS (2003) Validating Earth and ocean tide models using tidal gravity measurements. Geophys J Int 152:468-485

Bos MS, Scherneck HG (2014) Onsala space observatory. http://holt.oso.chalmers. se/loading. Accessed 13 July 2014

Boy JP, Gegout P, Hinderer J (2002) Reduction of surface gravity data from global atmospheric pressure loading. Geophys J Int 149:534-545

Calvo M, Hinderer J, Séverine R et al (2014) Time stability of spring and superconducting gravimeters through the analysis of very long gravity records. J Geodyn 80:20-33

Carrère L, Lyard F, Guillot A, Cancet M (2012) FES 2012: a new tidal model taking advantage of nearly 20 years of altimetry measurements. In: Proceedings of the 20 years of altimetry, Venice, Italy

Carrère L, Lyard F, Cancet M, Guillot A (2015) FES 2014: a new tidal model on the global ocean with enhanced accuracy in shallow seas and in the Arctic region. In: EGU general assembly conference abstracts, vol 17, p 5481

Chao B, Hsieh Y (2015) The Earths free core nutation: formulation of dynamics and estimation of eigenperiod from the very-long-baseline interferometry data. Earth Planet Sci Lett 36:483-492

Crossley DJ, Hinderer J (1995) Global geodynamics project-GGP: status report 1994. In: Poitevin C (ed) Proceedings of the workshop on non-tidal gravity changes. Luxembourg: Conseil de L'Europe Cahiers du Centre Européen de Géodynamique et de Séismologie, vol 11, pp 244-269

Cui XM, Sun HP, Rosat S et al (2014) Investigation of the time variability of diurnal tides and resonant FCN period. J Geodyn 79:30-38

Cui XM, Sun HP, Xu JQ et al (2018) Influence of core-mantle coupling on the excitation of free core nutation. Chin J Geophys 61(9):3584-3591 (in Chinese)

De Michelis P, Tozzi R, Meloni A (2005) Geomagnetic jerks: observation and theoretical modeling. Memorie Della Società Astronomica Italiana 76:957-960

Defraigne P, Dehant V, Hinderer J (1994) Stacking gravity tides measurements and nutation observations in order to determine the complex eigenfrequency of nearly diurnal free wobble. J Geophys Res 99:9203-9213

Dehant V, Laguerre R, Rekier J et al (2017) Understanding the effects of the core on the nutation of the Earth. Geodesy Geodyn 8(6):389-395

Ducarme B, Sun H, Xu J (2007) Determination of the free core nutation period from tidal gravity observations of the GGP superconducting gravimeter network. J Geod 81:179-187

Egbert GD, Erofeeva SY (2002) Efficient inverse modeling of barotropic ocean tides. J Atmos Ocean Technol 19(2):183-204

Hartmann T, Wenzel HG (1995) The HW95 tidal potential catalogue. Geophys Res Lett 22(24):3553-3556

Hinderer J, Legros H, Amalvict M (1982) A search for Chandler and nearly diurnal free wobble using Liouville equations. Geophys J R Astron Soc 71:303-332

Hinderer J, Boy JP, Gegout P et al (2000) Are the free core nutation parameters variable in time? Phys Earth Planet Inter 117:37-49

Lambert SB, Dehant V (2007) The Earth's core parameters as seen by the VLBI. Astron Astrophys 469:777-781

Malkin Z (2013) Free core nutation and geomagnetic jerks. J Geodyn 72:53-58

Mathews PM, Herring TA, Buffett BA (2002) Modeling of nutation and precession: new nutation series for nonrigid Earth and insights into the Earth's interior. J Geophys Res 107:539-554

Merriam JB (1992) Atmospheric pressure and gravity. Geophys J Int 109(3):488-500

Meurers B (2012) Superconducting gravimeter calibration by collocated gravity observations: results from GWR C025. Geophys J Int. https://doi. org/10.1155/2012/954271

Meurers B, Camp MV, Francis O et al (2016) Temporal variation of tidal parameters in superconducting gravimeter time-series. Geophys J Int 205:284-300

Olsen N, Mandea M (2007) Investigation of a secular variation impulse using satellite data: the 2003 geomagnetic jerk. Earth Planet Sci Lett 255:94-105

Roosbeek F, Defraigne P, Feissel M, Dehant V (1999) The free core nutation period stays between 431 and 434 sidereal days. Geophys Res Lett 26:131-134

Rosat S, Lambert SB (2009) Free core nutation resonance parameters from VLBI and superconducting gravimeter data. Astron Astrophys 503:287-291

Rosat S, Florsch N, Hinderer J, Llubes M (2009) Estimationof the free core nutation parameters from SG data: sensitivity study and comparative analysis using linearized least-squares and Bayesian methods. J Geodyn 48:331-339

Sun HP, Xu JQ, Ducarme B (2003) Experimental earth tidal models in considering nearly diurnal free wobble of the Earth's liquid core. Chin Sci Bull 48(9):935-940 (in Chinese)

Sun H, Cui X, Xu J, Ducarme B, Liu M, Zhou J (2009) Preliminary application of superconductive gravity technique on the investigation of viscosity at coremantle boundary. Chin J Geophys 52:311-321 (in Chinese)

Van Camp M, Vauterin P (2005) Tsoft: graphical and interactive software for the analysis of time series and Earth tides. Comput Geosci 31(5):631-640

Vondrák J, Ron C (2009) Stability of period and quality factor of free core nutation. Acta Geodyn Geomater 6(3):217-224

Vondrák J, Ron C (2010) Study of atmospheric and oceanic excitations in the motion of Earth's spin axis in space. Acta Geodyn Geomater 7(1):19-28

Vondrák J, Ron C (2015) Earth orientation and its excitations by atmosphere, oceans, and geomagnetic jerks. Serb Astron J 191:59-66

Wenzel HG (1996) The Nanogal Software: Earth tide data processing package ETERNA3.30. Bulletin d'Information de MareesTerrestres 124:9425-9439

Xu JQ, Sun HP (2009) Temporal variations in free core nutation period. Earthq Sci 22:331-336

Xu JQ, Sun HP, Ducarme B (2004) A global experimental model for gravity tides of the Earth. J Geodyn 38:293-306

Zhou YH, Zhu Q, Salstein D (2016) Estimation of the free core nutation period by the sliding-window complex least-squares fit method. Adv Space Res 57(10):2136-2140 